\title{
Coronary intervention
}

GW23-e0549 NEW-GENERATION DRUG-ELUTING STENTS IN PATIENTS WITH ACUTE MYOCARDIAL INFARCTION: A PROPENSITY SCORE MATCHED ANALYSIS

doi:10.1136/heartjnl-2012-302920l.1

${ }^{1}$ Kang-Yin Chen, ${ }^{2}$ Lin Wang, ${ }^{3}$ Yong-Jian Li, ${ }^{1}$ Guang-Ping Li, ${ }^{4}$ Seung-Woon Rha. ${ }^{1}$ Cardiology Department, The Second Hospital of Tianjin Medical University; ${ }^{2}$ Cardiology Department, Tianjin Chest Hospital; ${ }^{3}$ Cardiology Department, Tianjin Nankai Hospital; ${ }^{4}$ Korea University Guro Hospital

Objectives This study was aimed to compare everolimus-eluting stents (EES) with zotarolimus-eluting stents (ZES) in patients with acute myocardial infarction (AMI).

Background There is a paucity of data to exclusively evaluate the safety and efficacy of second-generation drug-eluting stents (DESs) in the setting of AMI.

Methods The present study enrolled 3309 AMI patients treated with ZES $(n=1608)$ or EES $(n=1701)$ in a large-scale, prospective, multicenter Korea Acute Myocardial Infarction Registry (KAMIR). Propensity score matching was applied to adjust for differences in baseline clinical and angiographic characteristics, producing a total of 2646 patients (1343 receiving ZES, and 1343 receiving EES). Target lesion failure (TLF) was defined as the composite of cardiac 


\section{ABSTRACTS}

death, recurrent nonfatal myocardial infarction (Re-MI), or target lesion revascularisation (TLR). Major clinical outcomes at 1 year were compared between the two propensity score matched groups. Results After propensity score matching, baseline clinical and angiographic characteristics were similar between the two groups. Clinical outcomes of the propensity score matched patients showed that despite similar incidences of Re-MI, in-hospital and 1-year mortality, patients in the EES group had significantly lower rates of TLF $(6.5 \%$ vs $8.7 \%, p=0.029)$, and probable or definite stent thrombosis $(0.3 \%$ vs $1.6 \%, p<0.001)$ as compared with those in the ZES group. Furthermore, there was a numerically lower rate of TLR $(1.2 \%$ vs $2.2 \%, p=0.51)$ in the EES group than in the ZES group.

Conclusions In this propensity-matched comparison, EES appears to be superior to ZES in reducing TLF and stent thrombosis in patients with AMI. 